Revue des patrimoines

46 | 2022

Le patrimoine de la Justice

\title{
La justice, un patrimoine muséal méconnu, l'exemple du musée de Bretagne
}

Justice in the Museum of Brittany's collections

\section{Laurence Prod'homme}

\section{(2) OpenEdition}

1 Journals

Édition électronique

URL : https://journals.openedition.org/insitu/33747

DOI : $10.4000 /$ insitu. 33747

ISSN : 1630-7305

Éditeur

Ministère de la Culture

Référence électronique

Laurence Prod'homme, «La justice, un patrimoine muséal méconnu, l'exemple du musée de Bretagne », In Situ [En ligne], 46 | 2022, mis en ligne le 12 janvier 2022, consulté le 03 février 2022. URL : http://journals.openedition.org/insitu/33747 ; DOI : https://doi.org/10.4000/insitu.33747

Ce document a été généré automatiquement le 3 février 2022

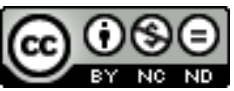

In Situ Revues des patrimoines est mis à disposition selon les termes de la licence Creative Commons Attribution - Pas d'Utilisation Commerciale - Pas de Modification 4.0 International. 


\title{
La justice, un patrimoine muséal méconnu, l'exemple du musée de Bretagne
}

Justice in the Museum of Brittany's collections

\author{
Laurence Prod'homme
}

1 Le musée de Bretagne, musée d'histoire et de société, labellisé musée de France, est intégré au sein de l'équipement culturel rennais «Les Champs Libres", qui regroupe également la bibliothèque métropolitaine et l'espace des sciences. Un parcours permanent d'exposition explore l'histoire de la Bretagne, des origines à nos jours, sur près de $2000 \mathrm{~m}^{2}$; une seconde exposition permanente est entièrement consacrée à l'affaire Dreyfus [fig. 1].

2 Le musée de Bretagne s'attache à mettre ses ressources à disposition de tous, à faciliter leur accessibilité et à donner des clés de lecture du monde actuel en faisant de ces collections une matière vivante.

3 Dans une telle configuration, on pourrait penser que les collections liées à la justice ne seront guère présentes et fort peu signifiantes. Pourtant, un fonds s'impose, celui lié à l'affaire Dreyfus: par sa constitution au fil des ans, par son utilisation comme ressources pour les chercheurs, par sa valorisation régulière auprès du public, il constitue une collection majeure sur une affaire dont les échos se font entendre aujourd'hui encore.

4 En 2019, la ville de Rennes, au travers d'un ensemble de manifestations regroupées sous l'intitulé «Affaire Dreyfus, Rennes se souvient et en parle », a souhaité réinterroger le procès en révision du capitaine Dreyfus ${ }^{1}$ et ses conséquences. Placée sous le haut patronage de Robert Badinter, la programmation a largement puisé dans les ressources du musée de Bretagne et a eu pour mission de rappeler, si besoin, combien cette décision judiciaire avait entrainé un cataclysme moral et politique dans de nombreuses sphères de la société française. 


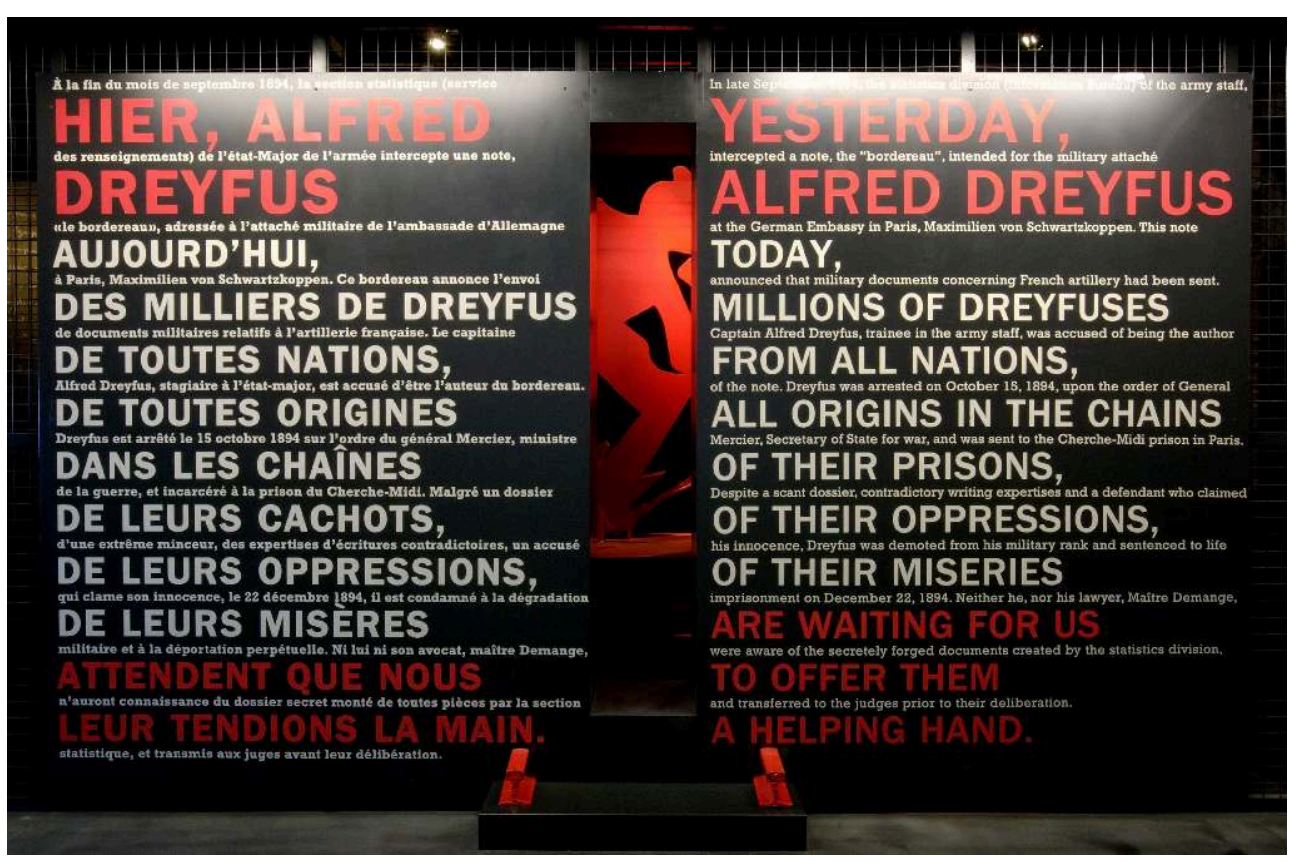

Entrée de l'exposition Dreyfus au musée de Bretagne, 2006.

(c) Alain Amet (musée de Bretagne).

Qu'en est-il des autres collections du musée davantage liées à la matière bretonne à proprement parler? Interrogeant la base de données du musée, la diversité des collections et le nombre de réponses pertinentes nous a presque surpris: si le parlement de Bretagne arrive bon premier, suivi par ceux qui l'ont fait vivre, avocats, parlementaires..., d'autres lieux où s'exerce la justice comme les tribunaux, les prisons, le bagne, constituent aussi des fonds significatifs. Dans un registre différent, des affaires criminelles ou encore des procès médiatisés ont donné lieu à toute une imagerie populaire ou à des reportages photographiques pour les plus récents. La période de la Seconde Guerre mondiale, tout comme l'après-guerre, ont produit, en Bretagne comme ailleurs, de nombreux documents, objets témoins d'une justice étroitement liée aux événements du moment.

6 Plus éclectique et diversifiée, en dehors du fonds Dreyfus très homogène, ce qui a trait à la justice, dans les collections du musée de Bretagne, témoigne largement de la vie des citoyens?, des diverses situations qui peuvent conduire chaque individu à y être un jour confronté.

\section{Le fonds Dreyfus : donner, valoriser, exposer}

7 La constitution du fonds Dreyfus au musée de Bretagne a la particularité de s'être enrichie au fil des années d'un perpétuel aller-retour entre des projets d'expositions et des dons. L'essentiel de la collection concerne la période du procès de Rennes mais comporte également quelques pièces antérieures ou postérieures; elle se caractérise par trois secteurs particulièrement bien représentés: la correspondance, l'iconographie et la presse. 
8 En 2020, on dénombre 7000 pièces dans la collection du musée de Bretagne, et si l'on y ajoute les périodiques et les quotidiens, le chiffre dépasse les 8000 items.

Le procès du capitaine Dreyfus s'est tenu au lycée de Rennes (actuel lycée Émile-Zola) durant l'été 1899, à proximité du musée des Beaux-Arts et d'Archéologie, les deux bâtiments se faisant face rue Toullier, rue par laquelle le public accédait à la salle d'audience.

10 Au moment du procès, un premier embryon de collection a été rassemblé par Lucien Decombe (1834-1905), alors conservateur du musée, probablement à titre de documents historiques : une série de cartes postales éditées par le libraire rennais Warnet-Lefèvre, quelques photographies et journaux, collection très réduite et inexploitée jusqu'aux années 1970.

11 C'est l'engagement d'un autre conservateur, Jean-Yves Veillard (1939-2020), qui favorise le développement de ce fonds : en 1973, il organise une première exposition intitulée "L'Affaire Dreyfus, une affaire toujours actuelle». Constituée en partie de prêts de documents originaux, cette exposition nourrit les premiers échanges fructueux qui vont s'instaurer et perdurer avec plusieurs membres de la famille Dreyfus.

12 Au cours des années 1970, il initie quelques dons assez modestes sur le procès de Rennes. L'année 1978 est marquée par le don décisif de Jeanne Lévy, fille de Lucie et Alfred Dreyfus : elle offre au musée plus de 4500 items, cartes postales, photographies, dessins, certaines pièces uniques et un ensemble exceptionnel de lettres et télégrammes adressés à ses parents. Cette démarche personnelle de Jeanne Lévy a un effet d'entraînement auprès d'autres membres de la famille ou de proches. C'est aussi à partir de cette date que le musée se porte acquéreur de collections complémentaires, achetées soit auprès de collectionneurs, soit en vente publique.

Le rôle de la famille Dreyfus dans l'enrichissement de cette collection a été moteur et fondateur, constant dans le temps, essentiel aussi quant au projet d'exposition permanente consacrée à « l'Affaire ».

14 Dans les pas de Jeanne Lévy, sa petite-cousine France Beck, petite-fille de Mathieu Dreyfus (frère aîné d'Alfred), procède entre 1978 et 1994 à pas moins de neuf dons et un dépôt, offrant ainsi au musée des photographies originales de grande qualité : portraits des parents d'Alfred Dreyfus, portraits de ses avocats, médailles, correspondance... et un manuscrit original d'Émile Zola actuellement conservé à la Bibliothèque nationale de France.

En parallèle, entre 1978 et 1999, le musée de Bretagne organise quatre expositions dont deux expositions itinérantes: la première, en 1978, circule dans toute la France pendant plus de dix ans et préfigure l'exposition présentée en 2019 à l'occasion des cent vingt ans du procès de Rennes. Au musée sont réalisées en 1994 "L'Affaire Dreyfus, l'arme du dessin »[fig. 2] et en 1999 «L'Affaire Dreyfus, un long combat contre l'injustice ». La Ville de Rennes, alors autorité de tutelle du musée de Bretagne, s'engage à travers la personne d'Edmond Hervé, maire de Rennes, à ouvrir une salle d'exposition permanente dédiée à l'affaire Dreyfus. 
Figure 2

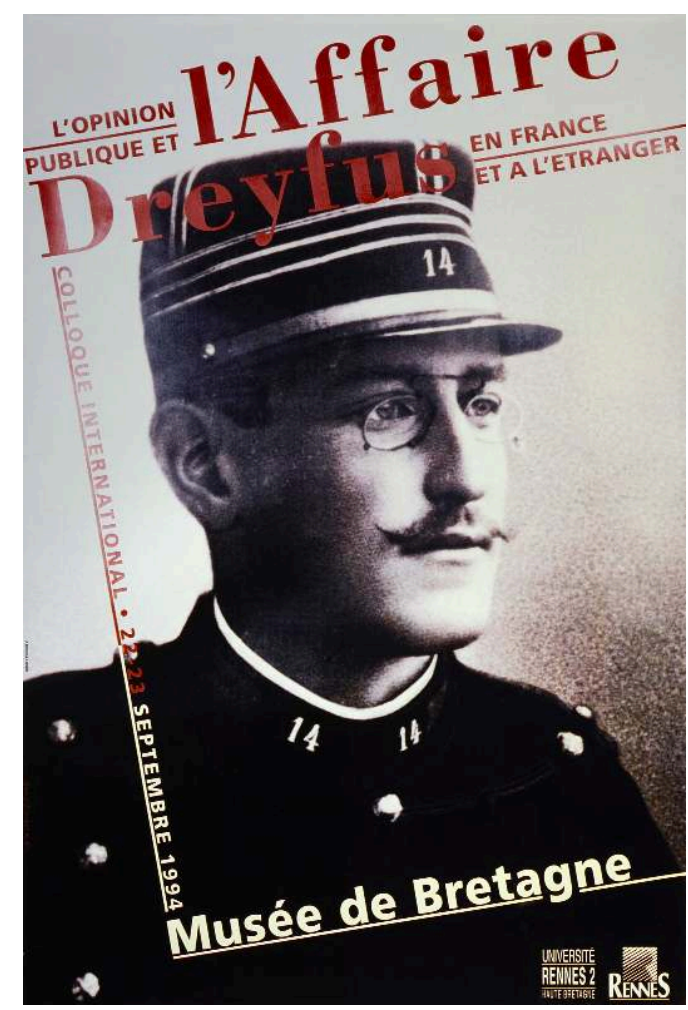

Affiche du colloque «L'opinion publique et l'affaire Dreyfus », réalisée par Alain Le Quernec et conservée au musée de Bretagne, 1994.

Reproduction Alain Amet (musée de Bretagne).

Au cours des années 1980, Ginette Zivy, nièce de Mathieu Dreyfus, confie au musée la magnifique série d'estampes de Charles Paul Renouard (1845-1924); l'historien américain Michael Burns donne à son tour un bel ensemble documentaire composé de coupures de presse et de correspondance, notamment de correspondance recopiée et contrôlée, échangée entre Lucie et Alfred.

Parallèlement, le musée poursuit ses acquisitions, et achète une collection de 376 cartes postales, parmi lesquelles les cartes que l'illustrateur Édouard Couturier (1871-1903) adresse avec de nombreux commentaires à Émile Straus (1865-1939). Ce dernier, journaliste, critique d'art, passionné par l'estampe, est le fondateur de la Société des collectionneurs de cartes postales illustrées. Dans la revue qu'il dirige sur ce sujet, il annonce une série de cinq cartes, peintes à la main qui, précise-t-il, «[sont] faites d'après d'excellents instantanés, se vendent, paraît-il, par milliers à Rennes [...]. Malgré les tirages successifs, il paraît que la série va être épuisée et elle commence à faire prime $^{2} »$.

18 Parmi les dons les plus remarquables au cours des années 1990, celui d'André Roumieux, dépositaire des archives d'Antoinette Scheurer, nièce d'Auguste ScheurerKestner (vice-président du Sénat et ardent défenseur de Dreyfus), révèle une correspondance particulièrement riche. Entre les années 1990 et 2020, le musée a réalisé de très nombreux achats, fréquemment soutenus par l'Association des amis du musée.

19 Concentrée essentiellement autour du procès de Rennes, la collection est très représentative de la production iconographique et de la surabondance d'images que 
suscita le procès. C'est un véritable déchaînement d'images qui investit la presse et construit, pour la première fois à cette échelle, un récit illustré d'une affaire juridique. Le public connaît la physionomie des acteurs principaux de l'Affaire et peut mettre un nom sur les visages. Les producteurs ou les utilisateurs de cette iconographie s'emploient eux-mêmes à la diffuser de toutes les façons possibles : en 1898, le critique John Grand-Carteret publie L'Affaire Dreyfus et l'image, un an plus tard Louis Rogès écrit Cinq semaines à Rennes, illustré de 200 photographies d'Aaron Gerschel, en 1903 est publié le Catalogue descriptif des cartes postales illustrées publié autour de l'Affaire... Émile Straus ne s'était pas trompé en écrivant: " Au point de vue politique, la carte postale sera un formidable adjuvant aux journaux illustrés satiriques et pamphlétaires ${ }^{3}$.»

Bien que les dons aient été moins nombreux durant ces vingt dernières années, récemment encore ce sont les arrière-petits-enfants du procureur Félix Froissart (1832-1924) qui ont offert au musée les treize volumes qu'il a lui-même soigneusement composés.

21 La constitution de la collection Dreyfus a, dès les années 1970, eu vocation à servir un projet d'exposition permanente. C'est en 2006, à l'ouverture des Champs Libres, que cet espace voit le jour. La conception scientifique en revient à Jean-Yves Veillard, accompagné pour la scénographie par l'architecte François Payet. Le choix fondamental de Jean-Yves Veillard a été de privilégier un parcours spectaculaire plus que linéaire ou simplement chronologique. Il a souhaité, pour développer ce parti-pris, une scénographie forte qui contribue à évoquer l'angoisse et la détresse du capitaine Dreyfus.

\section{Des mots et des images, une collection exceptionnelle}

\section{Écrire l'injustice, créer des « nœuds de vibration »}

Plus de 3000 lettres, télégrammes, cartes de correspondance font partie de la collection du musée ; parmi celles-ci, Gustave Schrumpf, de Wesserling en Alsace, écrit à Lucie Dreyfus en juin 1899 : «Chaque fois que j'avais pu gagner quelque nouvel adhérent à votre cause, j'en éprouvais un grand contentement. J'appelais cela des nœuds de vibration, ce qui faisait rire mon frère Christian Schrumpf, pasteur à Mulhouse, surnommé le "Capitaine Dreyfus" à cause de ses sentiments révisionnistes bien connus ${ }^{4}$.»

Quelle expression bien trouvée pour résumer la nature de cette immense correspondance! À la collection du musée, il faut ajouter les fonds de même nature conservés au musée d'Art et d'Histoire du judaïsme (Paris) ainsi que dans plusieurs services d'archives ou bibliothèques et fonds privés.

Cette abondante correspondance adressée au couple Dreyfus témoigne d'une connaissance pointue de l'Affaire dans le monde entier. À de très nombreuses reprises, les correspondants évoquent leur lecture assidue de la presse. Envoyées entre 1898 et 1901, le contenu et la tonalité de ces lettres varient selon les soubresauts de celle-ci : ainsi, lorsque la révision du procès est annoncée, les rédacteurs expriment leur optimisme et leur assurance quant à la réhabilitation de Dreyfus. Après le procès de Rennes, c'est l'effroi, l'indignation et l'incompréhension qui dominent. Souvent empreinte de lyrisme et d'emphase, cette correspondance dit beaucoup de l'empathie 
ressentie notamment à l'égard de Lucie, qualifiée d'« épouse admirable ", d'« héroïne courageuse ».

Bien que la presse soit le seul moyen d'information, on constate combien l'affaire Dreyfus a pénétré les foyers, et combien celles et ceux qui écrivent éprouvent dans leur chair le malheur des Dreyfus. Marceline Hennequin écrit dans ce sens à Dreyfus : «[...] il faut que vous ayez cette consolante pensée que vos souffrances ont fait naitre dans le cœur d'innombrables amis un courant d'estime, de sympathie, de vénérations tels que jamais personne n'a rien inspiré de semblable $[. . .]^{5}$.»

C'est un investissement et un attachement personnels qui les amènent à prendre la plume, car l'Affaire s'invite dans le quotidien familial, comme en témoigne cette Mme Jacques, qui écrit à Lucie: "Ce soir, en nous entendant parler, à table, de M. Dreyfus, mon petit garçon a eu un tel accès de larmes que nous ne pouvions plus le consoler $^{6}$. » Une autre femme, grand-mère de son état, écrit : «J'ai placé votre portrait et celui de vos enfants dans la chambre de mes petites-filles afin qu'elles aussi demandent à Dieu de ramener auprès de ces chers mignons leur père si longtemps absent $^{7}$.»

Venues de toute l'Europe et des Amériques, ces lettres affirment un engagement que l'on qualifierait aujourd'hui de " citoyen » : hommes et femmes, jeunes et vieux, de tous milieux, de toutes professions et de toutes confessions, affirment leur soutien indéfectible à Lucie et Alfred Dreyfus et leur soif de justice. Ancien lieutenant d'artillerie, E. Juillard écrit :

L'accusation tombait morceau par morceau. Aucune preuve, rien, rien, rien que des racontars de concierge dans l'ordre des faits et dans celui des préoccupations morales [...]. Quelles têtes de bois, quels cerveaux de plomb, quels cœurs secs, inaccessibles à toute émotion vraie, à toute pitié sincère, quels esprits [?] rebelles à l'évidence a donc fait à ces juges, braves soldats, hommes de bonne foi et d'intelligence dans les circonstances ordinaires de la vie, j'en suis sûr, l'odieuse campagne menée contre vous depuis deux ans pour les fins bandits du bas journalisme ${ }^{8}$.

S'il fallait rassembler les termes qui reviennent sans cesse à travers ces lettres, " courage ", « justice ", « innocence » et " martyr » se disputeraient la première place, exprimant ainsi le désarroi de tous ces épistoliers d'un jour, convaincus de l'innocence de Dreyfus et réclamant justice.

Bien qu'elle soit numériquement conséquente, et apporte un regard intime sur la perception de l'Affaire, cette correspondance ne représente qu'une partie de l'opinion ; elle ne doit pas faire oublier, au mieux l'indifférence, au pire la virulente opposition d'une large partie du public.

\section{Une affaire qui s'empare de l'image}

30 Cartes postales, estampes, affiches... toutes les techniques d'impression, tous les supports sont sollicités pour mettre en images l'affaire Dreyfus, la collection du musée s'en fait le témoin : 1554 cartes postales, éditées par quarante éditeurs différents, sont conservées au musée de Bretagne, et la collection, bien que non négligeable, n'est pas exhaustive. L'affaire Dreyfus en cartes postales constitue un vrai succès d'édition, pour un support alors encore jeune. En effet, c'est seulement à partir de novembre 1899 que 
les services postaux accordent officiellement l'autorisation d'utiliser la carte postale illustrée comme courrier ordinaire : la pratique a devancé la règle !

Les éditeurs ont vite perçu l'intérêt et le potentiel de diffusion de ces petits morceaux de carton illustrés : quelques éditeurs rennais, de nombreux éditeurs parisiens, mais aussi du monde entier se lancent dans la production de cartes postales. En Italie, en Allemagne, en Roumanie, aux Pays-Bas, en Suisse, en Hongrie... les éditeurs diffusent des séries thématiques autour de l'Affaire à Rennes. Le développement de la phototypie permet l'exécution technique de la carte postale illustrée et la photographie en constitue la source première ; on retrouve d'ailleurs les mêmes signatures sur les cartes postales et les photographies. Le phénomène est identique pour les cartes illustrées : illustrateurs et caricaturistes sont ceux qui exercent dans la presse illustrée et ils affichent pareillement leurs divergences d'opinion.

Les thèmes figurés témoignent des lieux importants du procès à Rennes: le lycée, naturellement, la gare, le logement de Lucie Dreyfus [fig. 3]... les portraits des principaux acteurs de l'Affaire sont nombreux; des séries de cartes illustrées par le dessin retracent l'histoire de l'Affaire depuis 1894. Des éditions de cartes postales illustrées sont encore réalisées en 1902, 1904, et jusqu'à la réintégration de Dreyfus dans l'armée, en 1906.

Figure 3

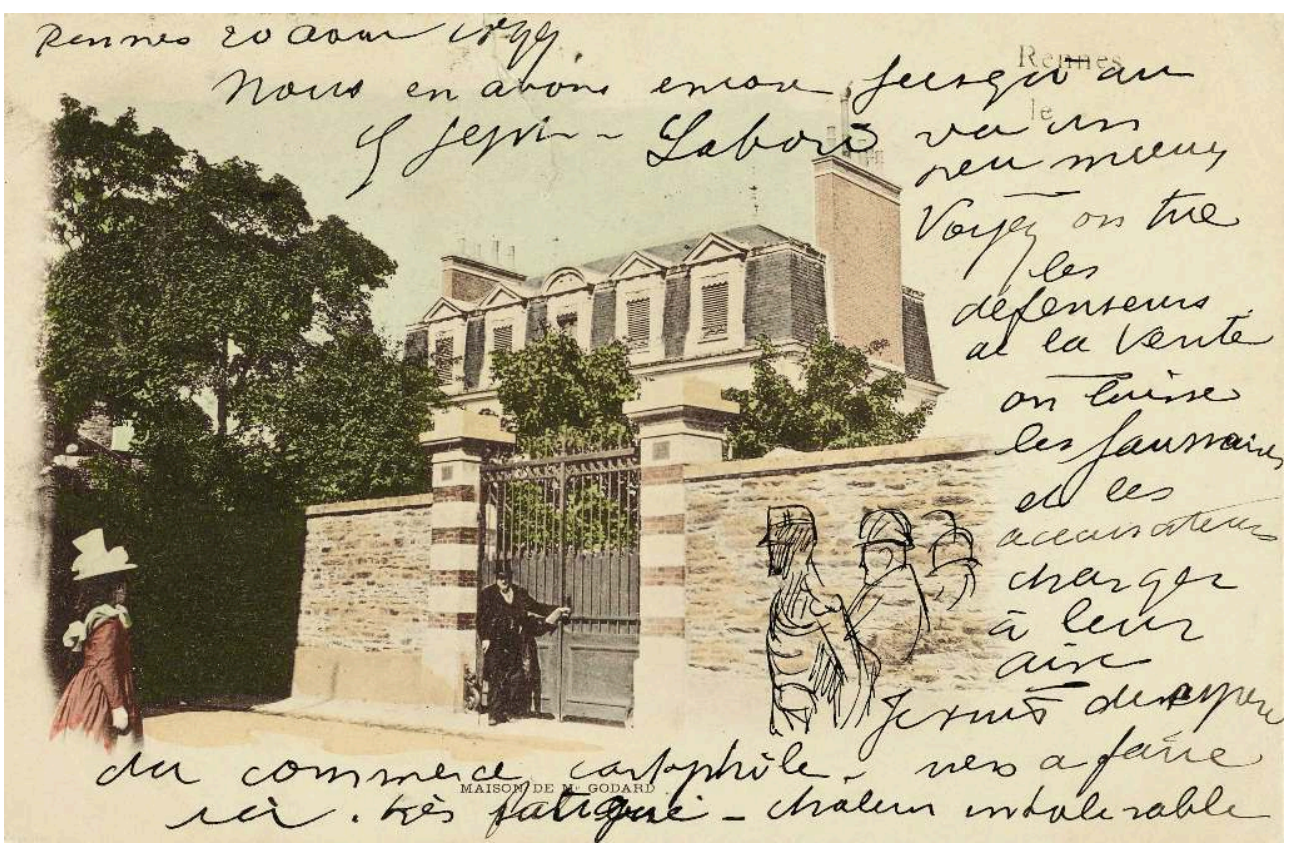

Carte postale figurant la maison de madame Godard où logeait Lucie Dreyfus, avec des annotations manuscrites évoquant l'attentat contre maître Labori. Carte adressée à Émile Straus, journaliste et critique d'art, le 20 août 1899, conservée au musée de Bretagne.

(c) Alain Amet (musée de Bretagne)

L'estampe, moyen plus traditionnel mais aussi plus qualitatif de reproduction, est présente dans les collections à travers la très belle série de Charles Paul Renouard : il collabore à de nombreuses revues en tant qu'illustrateur de presse. Ses dessins en noir et blanc sont très nombreux dans L'Illustration, la Revue illustrée ou encore dans les revues anglaises comme The Graphic ou Daily Graphic. Cette série, éditée d'après les dessins exécutés pendant le procès de Rennes, constitue un récit par la plume: 
certaines estampes très réalistes offrent une vision concrète et détaillée de la salle d'audience notamment, alors que de nombreux portraits témoignent d'un trait vigoureux qui campe la personnalité de chaque intervenant. La collection d'estampes rassemble également plusieurs portraits d'Émile Zola, ainsi qu'une série de tirés-à-part édités d'après des dessins caricaturaux. Une dizaine de chansons sur feuilles volantes, lithographies en noir et blanc ou en couleurs, évoquant l'Affaire ou plus largement, l'antisémitisme, sont aussi intégrées à cette collection.

Si l'Affaire trouve sa place dans les albums des collectionneurs, elle s'affiche aussi pour un plus vaste public sur les murs: le développement et le perfectionnement de la chromolithographie durant les dernières décennies $\mathrm{du} \mathrm{xIX}^{\mathrm{e}}$ siècle, ont permis à l'éditeur-imprimeur Victor Lenepveu de diffuser une suite de cinquante-deux affiches rassemblées sous le titre bien nommé de Musée des horreurs [fig. 4] : cette série atteint un sommet d'obscénité, de bestialité et d'antisémitisme rassemblés. En couleurs, ces affiches sont d'autant plus opérantes que le dessin y est remarquable de réalisme, et assure au premier regard l'identification des personnages. Victor Lenepveu avait en projet, après le Musée des horreurs, une seconde série intitulée Le Musée des patriotes, adressée gratuitement aux acheteurs de la première ; les cinq affiches connues à ce jour sont conservées dans nos fonds, elles mettent en avant les célébrités antidreyfusardes impliquées plus largement dans des mouvements nationalistes comme Henri Rochefort ou Édouard Drumont.

Figure 4

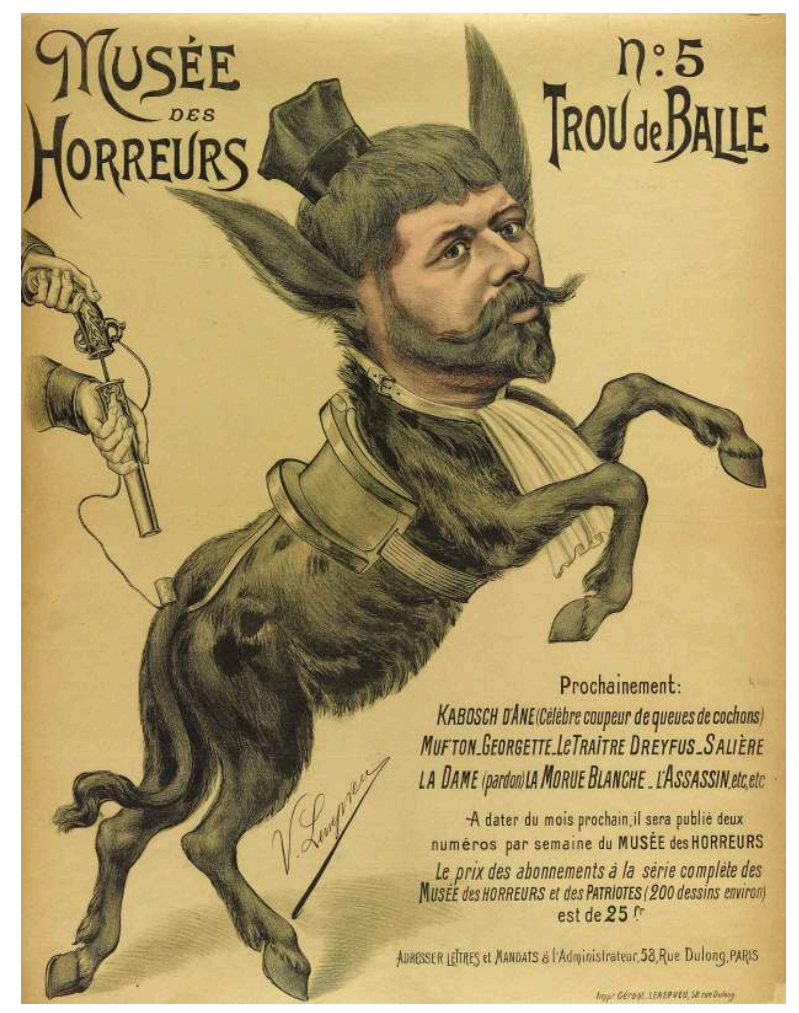

Affiche $n^{\circ} 5$ de la série du Musée des horreurs, Victor Lenepveu, vers 1899, conservée au musée de Bretagne. L'affiche montre l'un des deux avocats de Dreyfus, Fernand Labori, qui fut victime d'un attentat pendant le procès de Rennes.

(c) Alain Amet (musée de Bretagne). 

cette technique s'est avérée la plus efficace : le dessin de presse "témoin », fidèle et réaliste, et le dessin de presse engagé, pro- ou antidreyfusard, dont la ligne varie d'un trait réaliste à un trait caricatural. Le premier est le fruit d'une forte présence des dessinateurs judiciaires envoyés par les journaux illustrés comme témoins visuels des audiences : c'est le cas par exemple d'Édouard Couturier (1871-1903) qui, en quelques traits à peine esquissés, dresse des portraits vivants des protagonistes, saisissant avec brio postures et gestuelles. Dans un esprit plus naturaliste, les dessins de Louis Sabattier (1863-1935) [fig. 5] témoignent de l'atmosphère de la salle dans les moindres détails : l'histoire des trois dessins de Sabattier offerts par l'auteur au général Antoine Targe (qui joua un rôle actif dans la révision du procès, 1865-1942), puis remis par ses descendants au petit-fils de Dreyfus, Charles Dreyfus, qui en fit lui-même don au musée en 2002, ajoute à leur valeur historique une charge symbolique. 


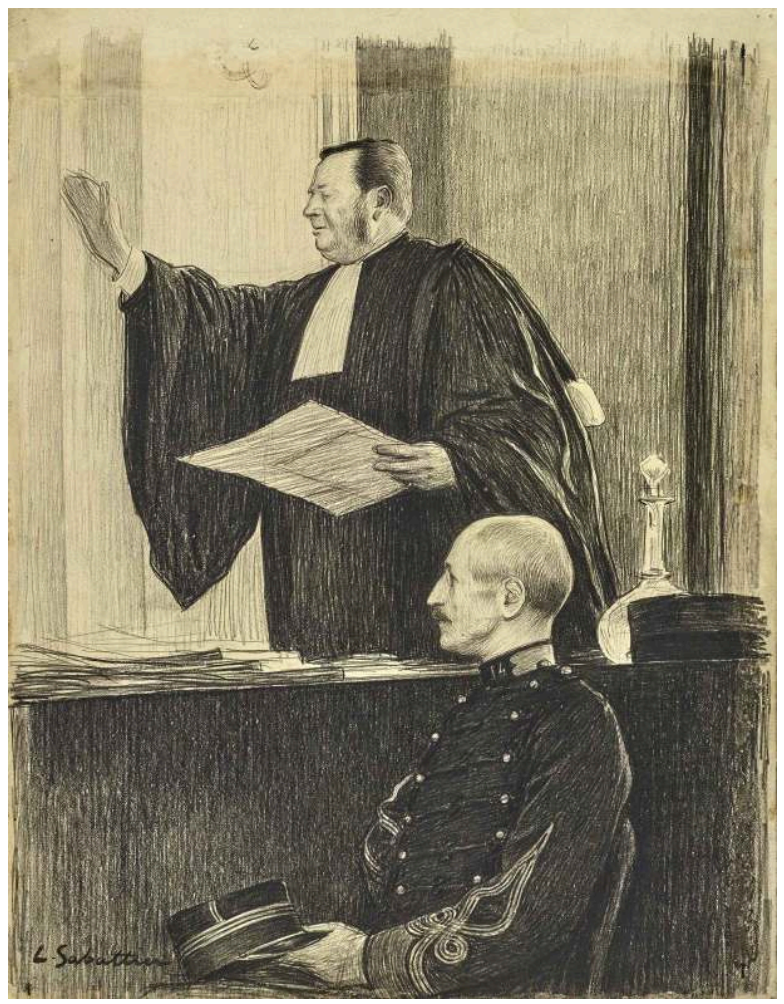

Alfred Dreyfus et maître Demange, Louis Sabatier, 1899, dessin publié dans la revue L'Illustration $n^{\circ} 2951$ du 16 septembre 1899 ; conservée au musée de Bretagne.

(c) Alain Amet (musée de Bretagne).

La seconde catégorie de dessins de presse, la plus abondante, se compose des dessins engagés, qu'ils soient ou non caricaturaux, d'ordinaire accompagnés d'une légende : trois dessins d'Henri-Gabriel Ibels (1867-1936), l'un des premiers adhérents à la Ligue des droits de l'homme, s'inscrivent dans ce groupe. Tous ont été publiés dans le journal qu'il fonda pour défendre Dreyfus, Le Sifflet. Dans le camp adverse, et dans la même veine que les dessins de Jean-Louis Forain (1852-1931) ou Caran d'Ache (1859-1909), le musée conserve une importante série de dessins satiriques d'Alfred Le Petit (1841-1909), qui publie dans Le Charivari et Le Grelot des caricatures violemment antisémites.

Du côté des journalistes, la photographie est devenue le nouvel outil indispensable. Plus de 400 photographies sont conservées dans les fonds du musée de Bretagne, dont beaucoup ont été publiées.

Un album mérite une attention particulière, il provient de la famille Hadamard, famille de Lucie Dreyfus, et retrace sous la forme d'un récit en images les moments forts du procès de Rennes. Les 205 photographies qu'il contient sont légendées, les personnages identifiés, quelques-unes portent la signature de Léon Bouët (1857-1911), d'autres le timbre sec d'Aaron Gerschel (1832-1910?), photographes très présents autour de l'Affaire.

42 Les photographies étant interdites à l'intérieur de la salle d'audience, une grande majorité a été prise dans la cour du lycée, cour dans laquelle défenseurs et détracteurs échangent, fument et patientent lors des interruptions de séance. Louis Rogès témoigne de leur présence: "[Les photographes] surgissent de partout, amateurs et 
professionnels. Les uns munis d'appareils savants, de lorgnettes compliquées, les autres simplement d'un petit kodak de poche ${ }^{9}$.» Malgré le grand nombre de photographes présents, les prises de vue sont toutes très proches et offrent des images récurrentes. De rares photographies plus originales figurent les journalistes, ou encore maître Edgar Demange, défenseur de Dreyfus, lisant son journal au café.

Trois photographies signées Valerian Gribayedoff (1858-1908) constituent les uniques clichés connus à ce jour et pris à l'intérieur de la salle d'audience, très probablement le premier jour du procès : au premier plan les journalistes, sur la droite Alfred Dreyfus et ses avocats. Une centaine de photographies prises en studio, par Gerschel, Nadar, Pierre Petit, Léopold-Émile Reutlinger... studios parisiens ayant pignon sur rue, constituent une galerie de portraits de la grande majorité des acteurs de l'Affaire.

\section{De la réclame à la commémoration}

Pour achever ce tour d'horizon des collections liées à l'affaire Dreyfus, on ne peut omettre les collections d'objets qui sont de nature très variée.

Objets commémoratifs ou objets "publicitaires » ont vocation à transmettre un message politique et un engagement fort pour ou contre Dreyfus. Les médailles, médaillons [fig. 6] et insignes constituent une série importante d'objets réalisés dans des matériaux très divers, du plus luxueux au plus ordinaire ; en 1906 encore, alors que Dreyfus est sur le point d'être réhabilité, une médaille à l'effigie du général Auguste Mercier, ministre de la Guerre à l'origine de l'Affaire, réaffirme les positions de ce dernier.

Figure 6

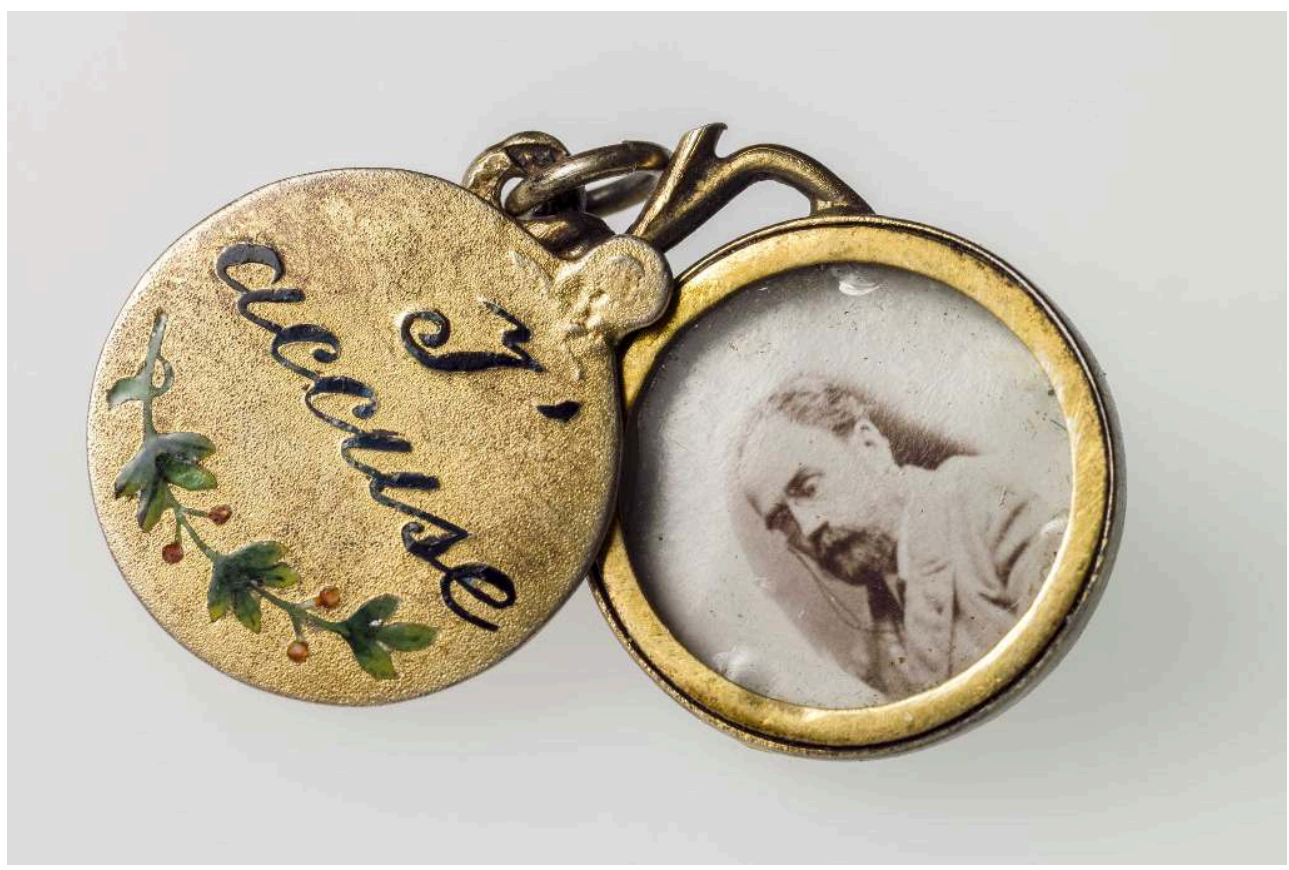

Médaillon contenant un portrait d'Émile Zola, et portant l'inscription « J'accuse », en référence à la lettre de l'écrivain parue dans L'Aurore du 13 janvier 1898. Médaillon conservé au musée de Bretagne. (c) Alain Amet (musée de Bretagne). 
46 catégorie d'objets. Plusieurs sculptures attestent de l'impact de l'Affaire auprès des artistes : modèle réduit de la statue de Tim (Louis Mittelberg, 1919-2002) Hommage au capitaine Dreyfus (1985; boulevard Raspail, Paris), buste d'Émile Zola par Alexandre Zeitlin (1872-1946), Le Crieur de journaux par F. Gilles (d'après le sculpteur José de Charmoy, 1879-1914) ou encore imposante sculpture en acier La dégradation de Dreyfus $^{10}$ (1988) offerte au musée par l'artiste Igaël Tumarkin (né en 1933).

Dans un autre registre, la « réclame » investit des supports pour le moins surprenants, comme ces étuis de papier à cigarette, dénommé « le papier du bordereau », qui, d'un étui à l'autre, relate les grandes étapes de l'Affaire. Les images à collectionner déferlent en quantité impressionnante dans les dernières décennies du XIX ${ }^{e}$ siècle et l'Affaire est un sujet dont s'emparent les marques de chocolat, de chicorée, les grands magasins, etc.

Entre ces deux catégories, dans un registre « ludique » se trouvent différents jeux, type «jeu de l'oie», images animées ou marionnettes, à l'allure toujours féroce et caricaturale pour les antidreyfusards, simplement réalistes dans l'autre camp.

La collection Dreyfus du musée de Bretagne s'enrichit régulièrement de nouvelles acquisitions. En 2019, deux dessins d'Henri-Gabriel Ibels ont été achetés, dont l'un publié en couverture du Sifflet, journal qu'il avait fondé pour la défense de Dreyfus et pour lequel il réalisa de très nombreuses unes pendant toute la durée du procès.

Publié dans Le Sifflet du 8 septembre 1898, il fait référence au procès d'Émile Zola. L'attitude d'Albert Delegorgue, président de la cour d'assises, est restée dans les annales par sa volonté réitérée de limiter la durée et la nature des débats. Il refusa toute évocation de l'affaire Dreyfus, coupant systématiquement la parole à maître Fernand Labori. Sa fameuse phrase : «Non, la question ne sera pas posée! » [fig. 7] fut répétée à maintes reprises et moquée par le clan des dreyfusards. La légende du dessin indique qu'elle fut finalement posée et mit en difficulté les représentants de l'ÉtatMajor, particulièrement le lieutenant-colonel Hubert Henry, qui y répondit en se suicidant. 
Figure 7

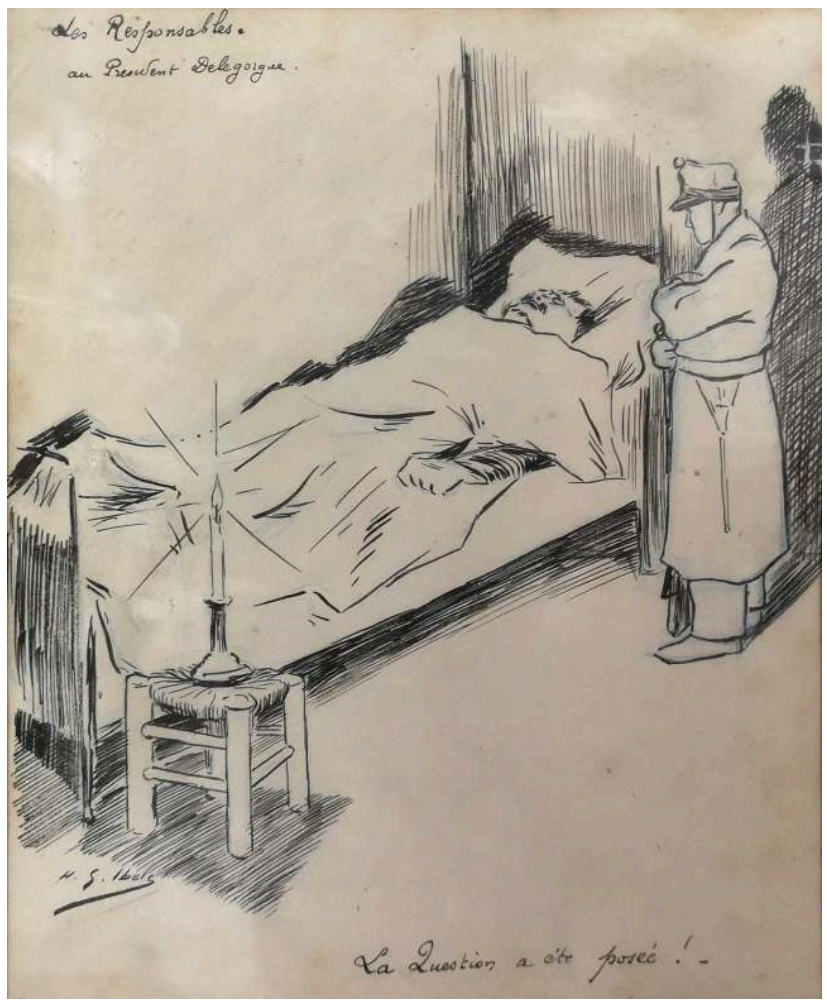

La question a été posée !, Henri Gabriel Ibels, 1898. Dessin conservé au musée de Bretagne.

(c) Alain Amet (musée de Bretagne).

\section{Du patrimoine bâti aux acteurs de la justice}

Passer d'une collection Dreyfus, constituée dans une unité de temps et autour d'une thématique restreinte, au thème plus large de la justice dans les collections n'est pas un exercice aisé ! Difficile d'éviter l'inventaire à la Prévert, en prenant l'abécédaire dans le désordre, avocat, tribunal, prison, jugement, procès... De nombreuses thématiques peuvent se rattacher au sujet, en se positionnant sur une chronologie beaucoup plus élastique et très hétérogène.

\section{Des lieux pour dire la justice}

Le patrimoine bâti nous semble un axe particulièrement bien représenté, à commencer par le parlement de Bretagne à Rennes. Bâtiment majeur du patrimoine architectural rennais, nos prédécesseurs ne s'y sont pas trompés qui, dès le xix ${ }^{e}$ siècle, lui ont donné une place de choix. La place et le bâtiment se confondent fréquemment, comme dans cette vue cavalière de 1644 intitulée Ville épiscopalle, siège du parlement et capitalle du duché de Bretagne. Établi à Rennes depuis 1561, le parlement de Bretagne associe cour de justice et organe de gestion administrative de la province. Au cours du XVIII ${ }^{e}$ siècle, plusieurs estampes, dont celle éditée chez Basset rue Saint-Jacques à Paris figurent justement le parlement encadré par les imposants immeubles qui bordent la place. Cette estampe titrée Vue perspective de la nouvelle Place du Palais de Rennes [fig. 8] met en avant la place du Parlement comme lieu de promenade pour l'aristocratie. Les 
collections témoignent des variations architecturales du parlement, à plusieurs reprises remanié, notamment suite à l'incendie de 1720, autant qu'aux usages qui eux aussi fluctuent selon les époques. Deux séries de dessins d'architecte évoquent les travaux intérieurs successifs du xIx siècle: au cours des années 1830, François Toussaint de Lagarde exécute un ensemble de plans matérialisant divers travaux d'amélioration; à peu près à la même période, Louis-René Richelot (1786-1855) réalise des plans destinés à des travaux de chemisage. Quelques estampes des dernières décennies du XIXe siècle valorisent la place et le parlement, très vite suivies par des photographies en grand nombre, qui ne manquent pas de mettre en avant les décors intérieurs.

Figure 8

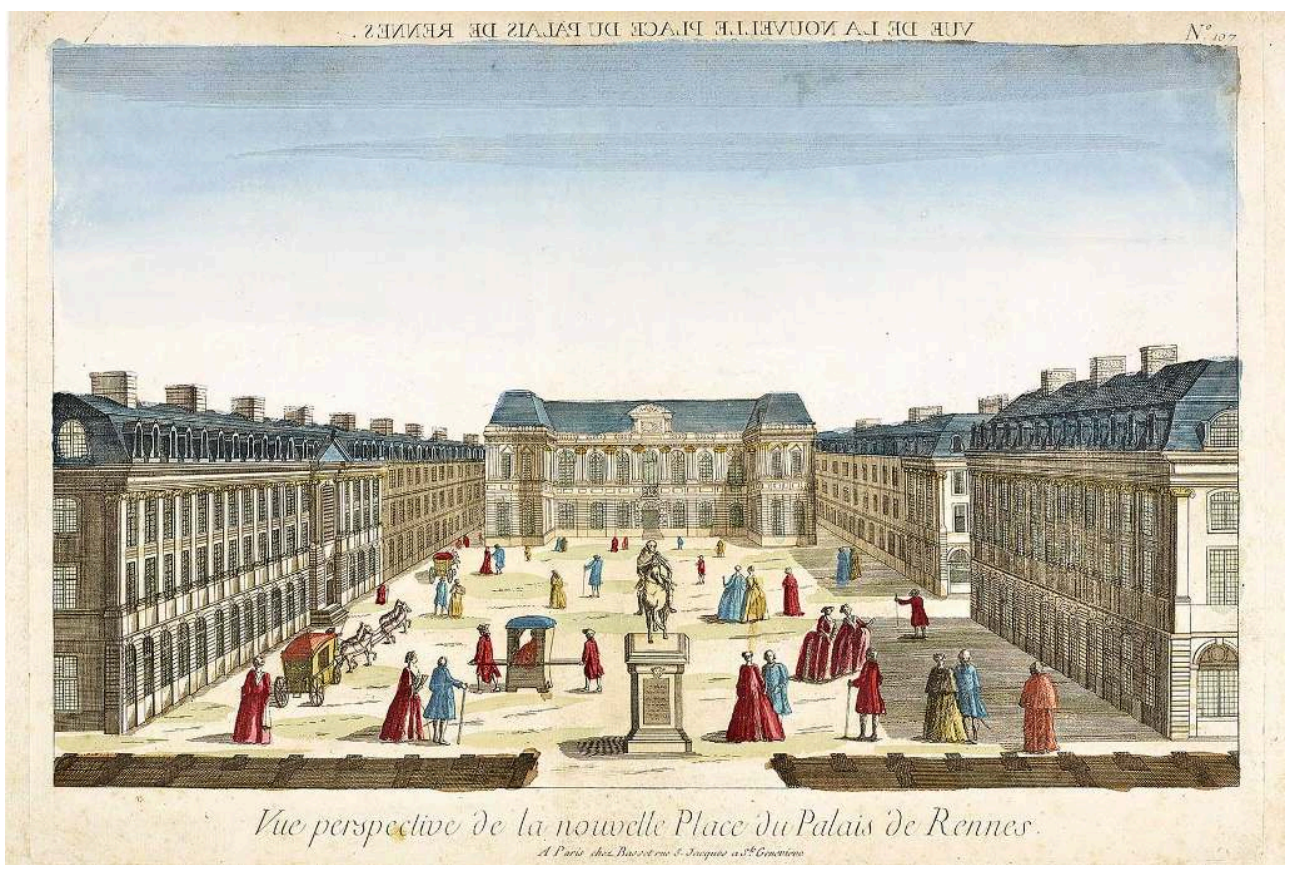

Vue perspective de la nouvelle Place du Palais de Rennes, estampe, Basset éditeur, rue St Jacques à Paris, avant 1726.

Reproduction musée de Bretagne

Les vues du bâtiment, extérieures comme intérieures, sont aussi convoquées pour signifier les usages du lieu et ses acteurs : en 1690, à l'occasion du retour du parlement à Rennes après un exil forcé de quinze ans à Vannes, est éditée une estampe évoquant l'événement. Louis XIV d'un côté, la Bretagne accompagnée des allégories de la justice et de la vertu de l'autre, posent devant le palais. Au pied de l'escalier monumental, un groupe d'hommes, dont un magistrat tenant dans sa main un sac de procédures. Le musée possède trois sacs de ce type, en toile de jute : ils servaient à conserver, jusqu'à la Révolution, toutes les pièces d'un procès; sur chaque sac est fixé un petit papier résumant l'affaire concernée.

Les parlementaires sont extrêmement présents dans les collections, par le biais de portraits gravés ou peints, mais aussi par des objets personnels comme des pièces d'orfèvrerie ou de céramique portant les armes de leur famille. Pour le XviII ${ }^{\mathrm{e}}$ siècle sont conservés quelques objets significatifs comme une série de sceaux, un banc portant l'inscription "Pour Messieurs du parlement », une garniture de chaise réalisée par la 
manufacture des Gobelins pour la grande chambre ou encore une très belle bourse brodée aux armes des États de Bretagne [fig. 9].

Figure 9

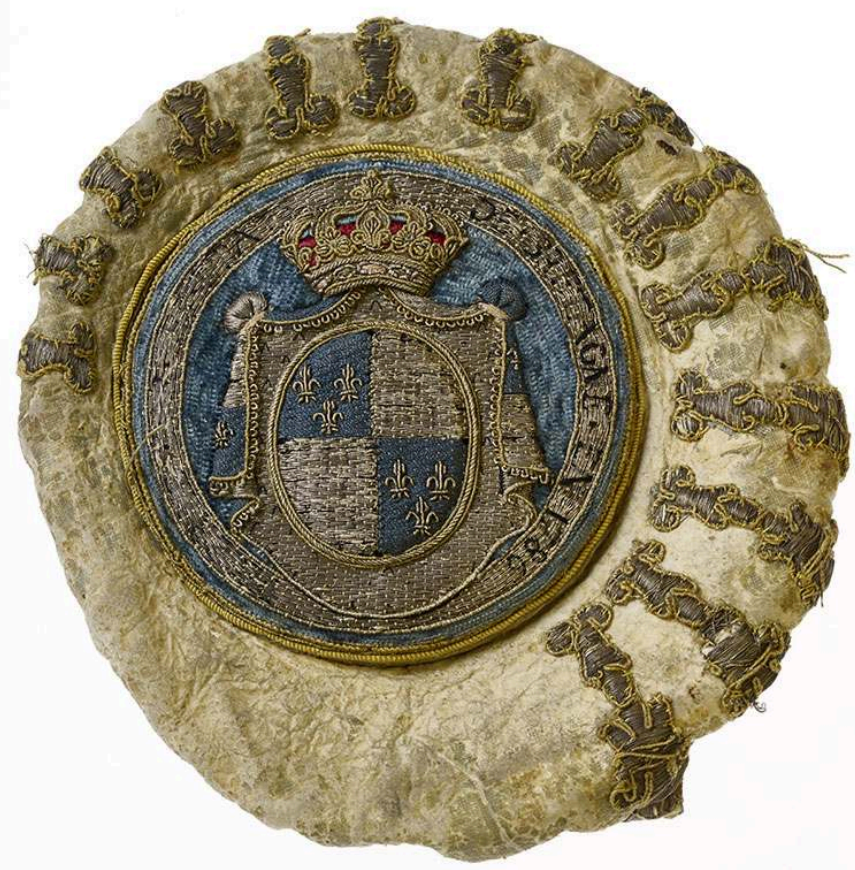

Bourse de jetons ou de gratification, 1786, conservée au musée de Bretagne.

(c) Alain Amet (musée de Bretagne).

Enfin, on ne peut passer sous silence la place symbolique du parlement dans le cœur des Rennais : c'est sur la place que se déroulèrent pendant très longtemps fêtes civiles ou militaires, c'est un lieu de promenade et de loisirs, on ne compte plus les productions imprimées qui utilisent le bâtiment comme motif principal. C'est le bâtiment phare de la ville et l'incendie de 1994 a conforté cet attachement.

Le poids écrasant du parlement de Bretagne ne doit pas faire oublier les nombreuses collections figurant des bâtiments où est rendue la justice ou sont appliquées ses décisions, sur l'ensemble du territoire de la Bretagne. Les collections d'estampes, de dessins, de photographies et de cartes postales constituent une source iconographique importante: les tribunaux locaux, les prisons, le bagne de Brest, les centres de rééducation de Dinan, Belle-île-en-Mer ou Rennes sont très bien représentés dans les fonds. Citons pour exemple la prison centrale des femmes [fig. 10], sur laquelle l'architecte Alfred Normand (1822-1909), qui pratiquait également la photographie, a réalisé en 1887, vingt ans après son achèvement, un reportage assez exceptionnel. Les objets, bien que rares, ne sont pas totalement absents : menottes et entraves provenant des cachots du château de Vitré, ou encore uniforme de bagnard [fig. 11] provenant du bagne de Cayenne. 
Figure 10

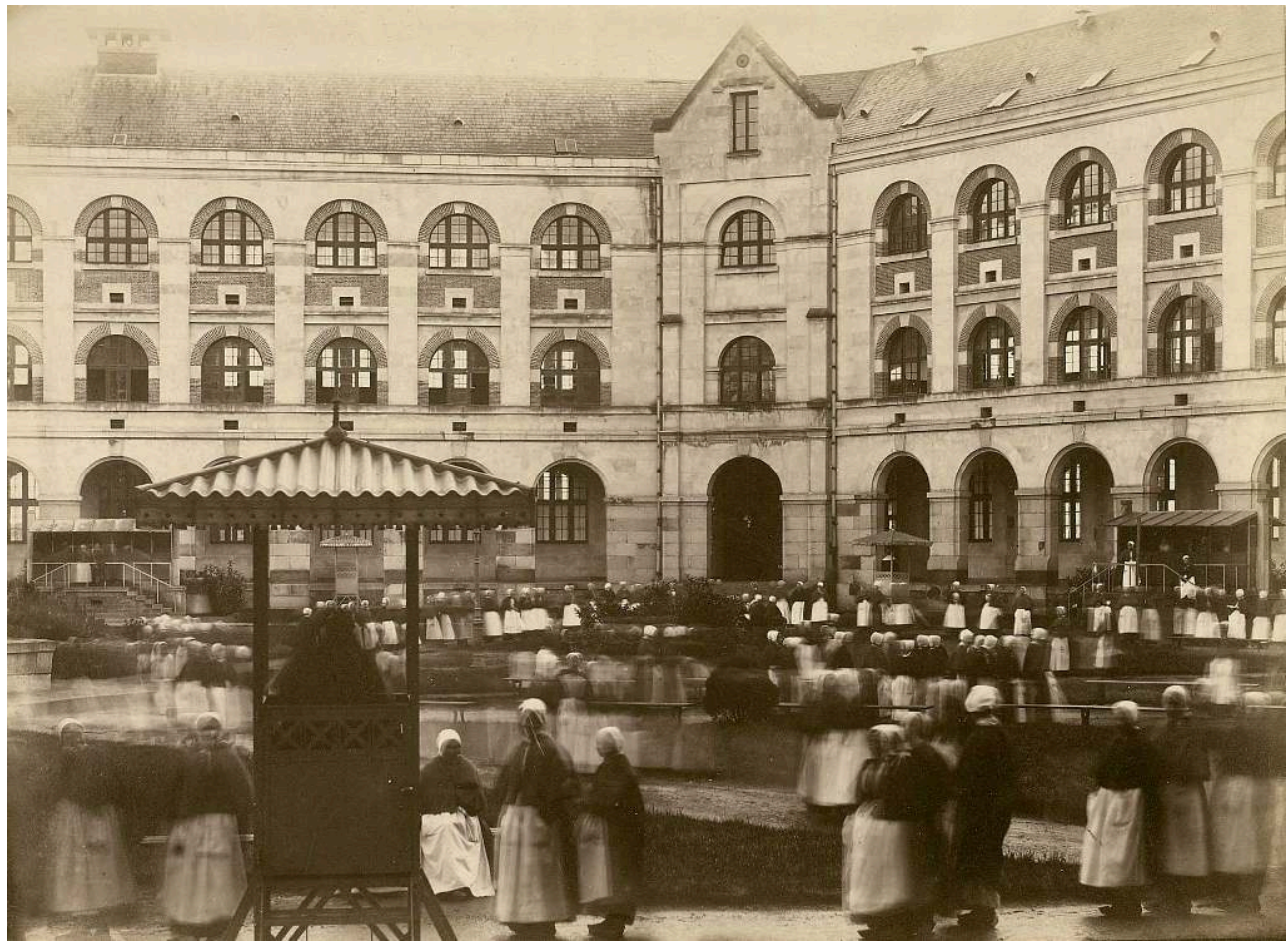

Rennes maison centrale des femmes, I'heure de la promenade, Alfred Normand, 1887, photographie conservée au musée de Bretagne.

(c) Alain Amet (musée de Bretagne). 
Figure 11

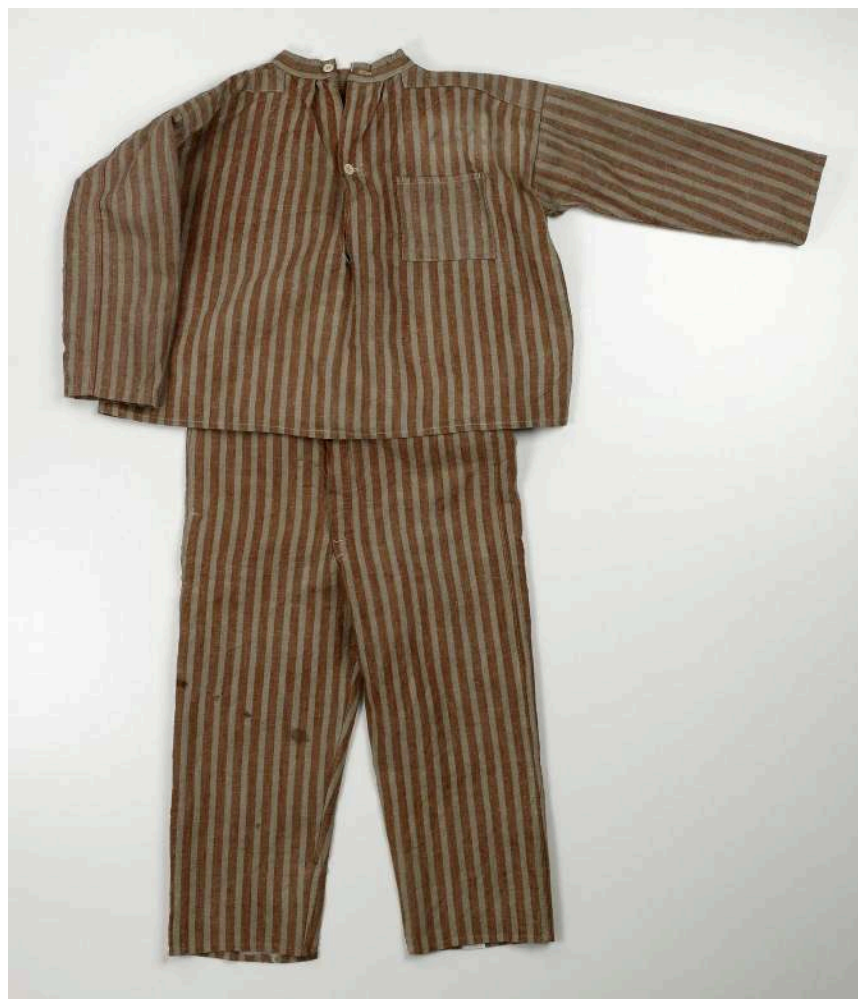

Costume de bagnard, toile de lin, porté à Cayenne, début du 20e siècle, conservé au musée de Bretagne.

(c) Alain Amet (musée de Bretagne).

\section{Portraits des acteurs}

57 Parmi les acteurs de la justice, magistrats, avocats et juges sont très présents dans les collections : des estampes du XIX ${ }^{e}$ siècle figurant le président de la cour impériale ou le doyen de la faculté de droit, en passant par les images populaires ou statuettes à l'effigie de saint Yves, patron des professions liées à la justice, l'iconographie abonde et se montre parfois caricaturale. Pour les périodes plus récentes, le fonds du photographe Sigismond Michalowski (1925-2003) reçu en 1986 contient de nombreux reportages effectués dans les années 1980 sur la cour d'appel de Rennes [fig. 12], l'installation du procureur ou encore des portraits individuels de magistrats. Une robe et toque d'avocat figurent aussi dans les registres d'inventaire de l'année 1933, plus récemment une robe de conseiller à la cour d'appel de Rennes a fait son entrée dans les collections à l'occasion d'une collecte sur le thème des vêtements professionnels. 
Figure 12

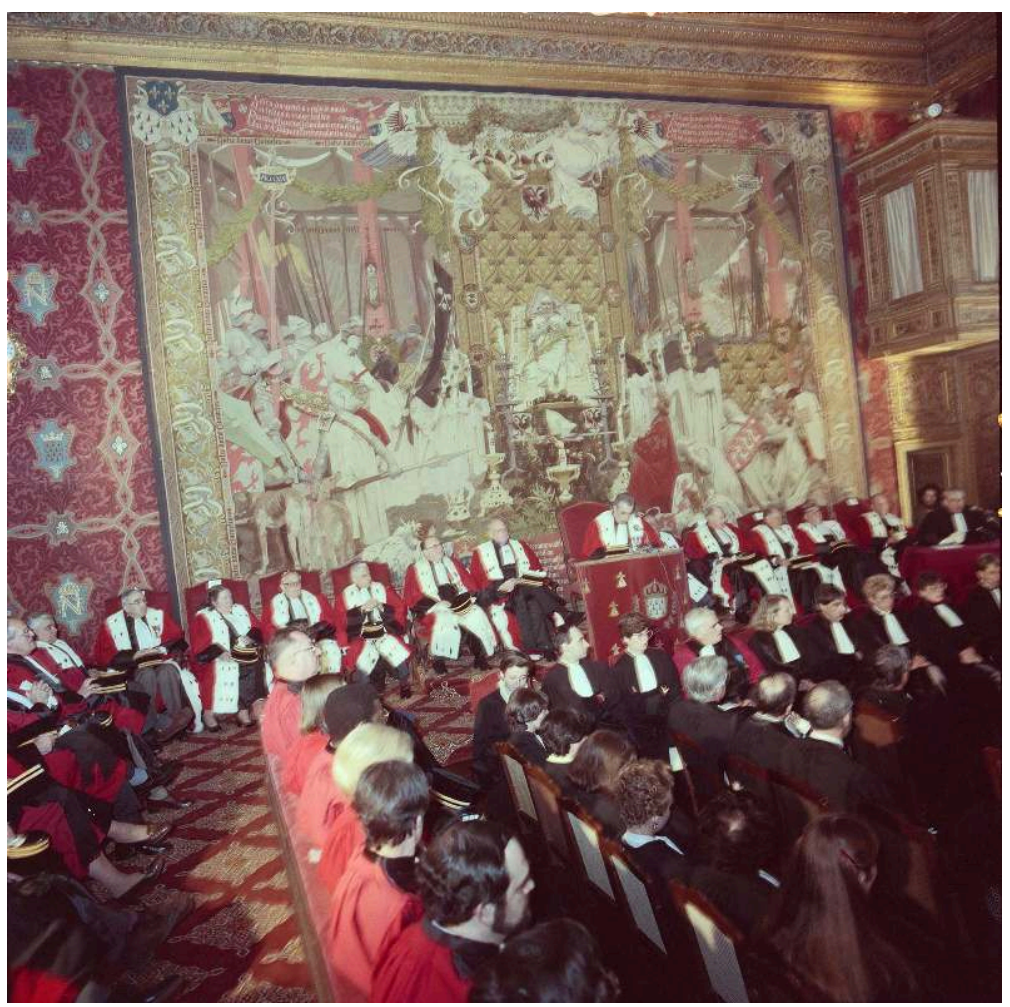

Rentrée de la cour d'appel de Rennes, janvier 1985, négatif photographique conservé au musée de Bretagne.

(c) Sigismond Michalowski / Rennes métropole.

Le thème de la criminalité, associé à celui des procès qui potentiellement en découlent, constitue l'un des derniers sujets bien présent à travers les collections du musée de Bretagne. Le XIX ${ }^{\mathrm{e}}$ siècle a beaucoup exploité ces thématiques diffusées par le biais de l'image populaire et de la chanson sur feuille volante, puis repris par la presse, notamment à travers les romans-feuilletons. Les meurtres perpétrés par la célèbre empoisonneuse Hélène Jégado ${ }^{11}$ (1803-1852), dont le musée conserve aussi le masque mortuaire, ont fait l'objet d'images populaires, mais des crimes moins spectaculaires liés à des événements locaux ont aussi été diffusés par ce biais. Le fonds d'images populaires étant très conséquent, celles illustrant des faits qui se sont déroulés en dehors de la région comme l'assassinat du président Sadi Carnot (1894) ou l'affaire du crime de Pantin (1869) ont aussi leur place dans les collections.

Pour le $\mathrm{xx}^{\mathrm{e}}$ siècle, ce sont les photographies qui illustrent ces thématiques, avec là aussi un volet régional, comme après-guerre les procès de la collaboration, et un volet national comme les procès du juge Henri Pascal en 1975 ou celui de Gérard Nicoud en 1986, l'un s'étant déroulé à Rennes, l'autre à Quimper. Les mouvements pour l'autonomie de la Bretagne se réclament d'une justice régionale ; les tracts [fig. 13] ou les affiches produits dans ce contexte se réfèrent notamment au parlement de Bretagne. 
Figure 13

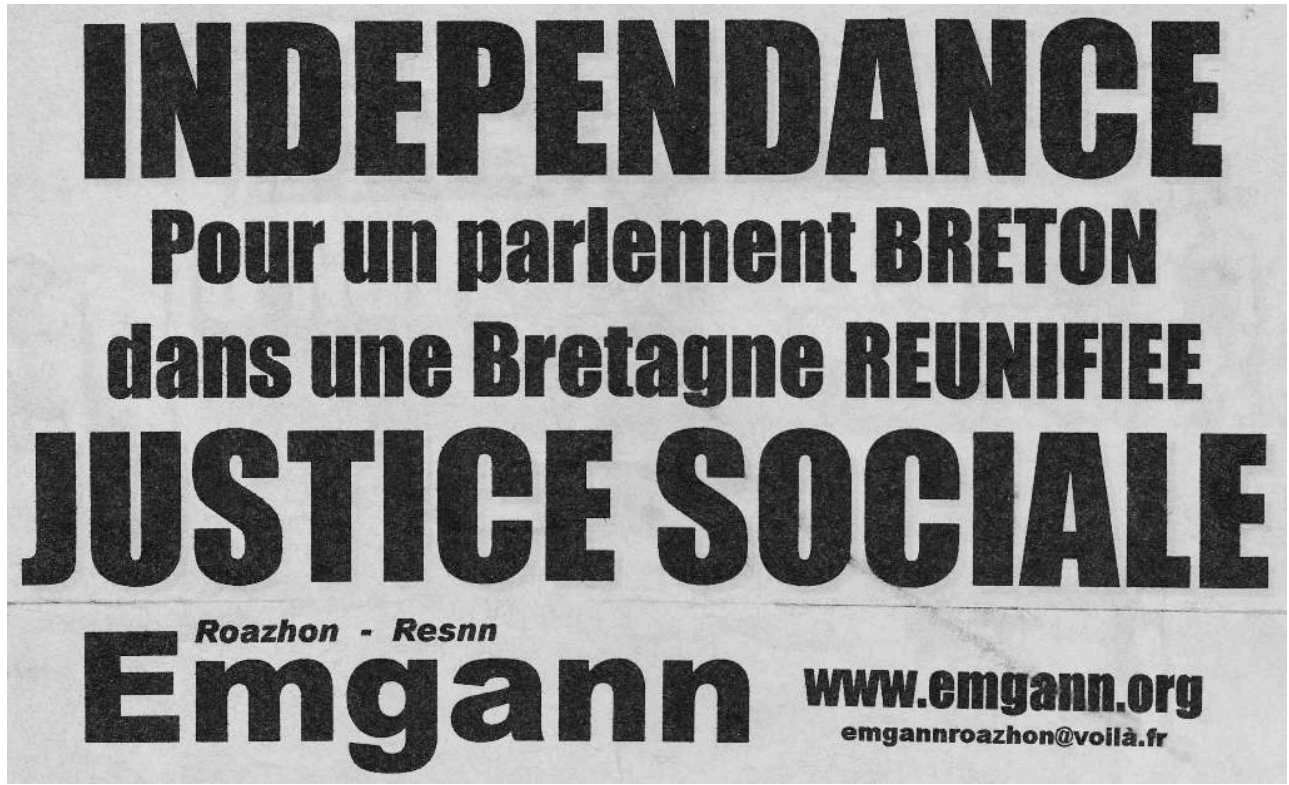

Tract de propagande pour le mouvement Emgann, années 1980, conservé au musée de Bretagne. (c) Alain Amet (musée de Bretagne).

Une autre forme de justice est également revendiquée par les citoyens, la justice sociale: elle traverse de manière régulière l'ensemble des collections, même si ces dernières sont plus fournies pour le $\mathrm{xx}^{\mathrm{e}}$ siècle. Les fonds photographiques illustrent en particulier toutes les luttes sociales [fig. 14] marquées par de nombreuses manifestations au cours des cinquante dernières années.

Figure 14

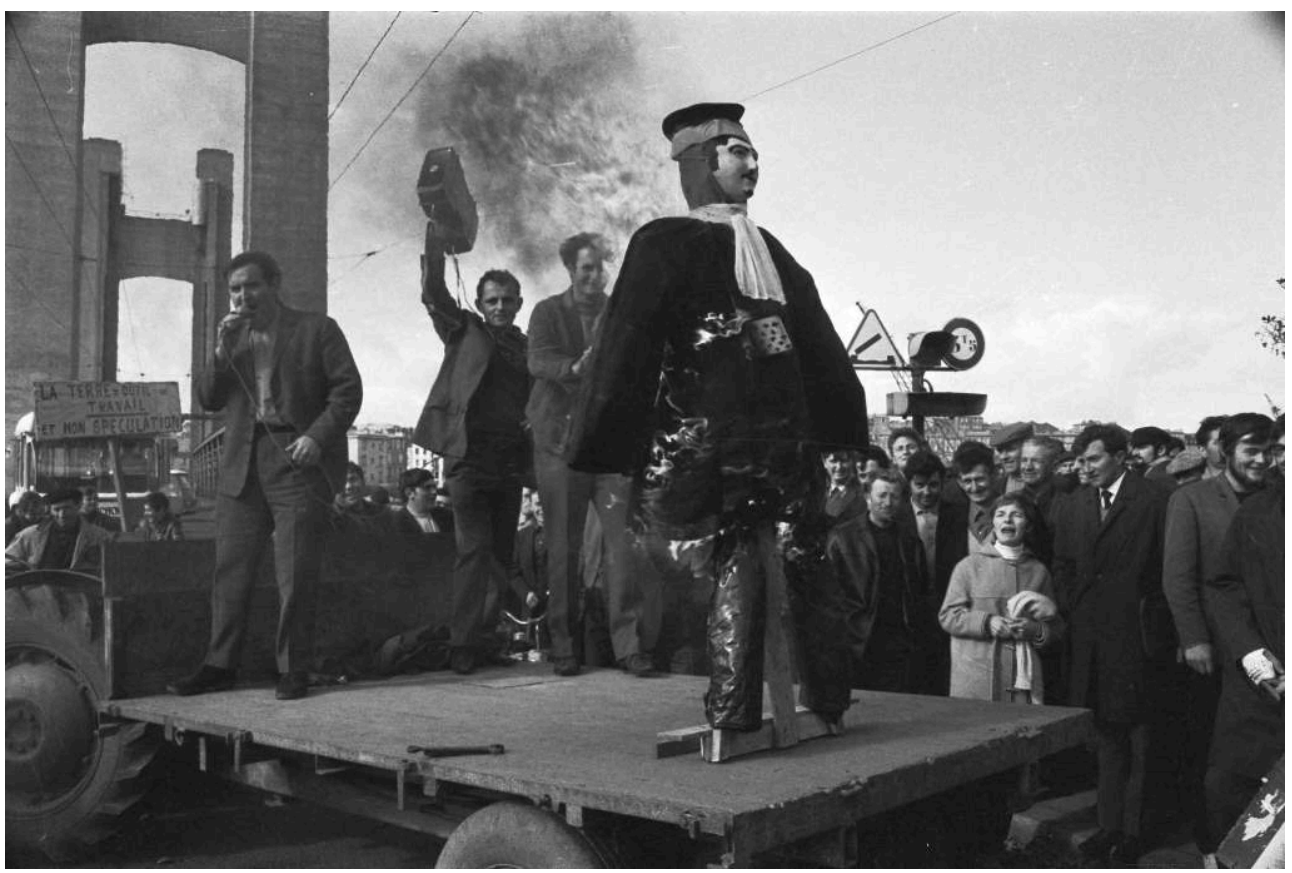

Manifestation d'agriculteurs, Brest, années 1960, négatif photographique conservé au musée de Bretagne.

(c) Sigismond Michalowski / Rennes métropole. 
61 À l'exception du fonds Dreyfus dont l'ampleur était cernée, nous n'aurions pas a priori, et avant de questionner le sujet, pensé trouver autant de collections qui pouvaient rentrer dans la thématique justice.

62 Certes, l'iconographie domine mais les collections d'objets ne sont pas absentes pour autant: d'autres items, non signalés ici, auraient pu être cités pour évoquer ce sujet ; nous invitons les lecteurs à en faire eux-mêmes la découverte en parcourant le portail des collections du musée de Bretagne et en l'enrichissant au besoin de leurs connaissances $^{12}$.

\section{NOTES}

1. Le procès en révision du capitaine Dreyfus s'est tenu à Rennes du 7 août au 9 septembre 1899.

2. "L'événement cartophilique du mois", L'Amateur de la carte postale illustrée, $\mathrm{n}^{\circ} 3$, aoûtseptembre 1899 ,

p. 7

3. GRANOUX Xavier, "L'Affaire Dreyfus", catalogue descriptif des cartes postales illustrées françaises et étrangères parues depuis 1894, Paris, H. Daragon, 1903, p. 1.

4. Voir http://www.collections.musee-bretagne.fr/ark:/83011/FLMjo153540 [lien valide en octobre 2021]. Lettre du 9 juin 1899, collection musée de Bretagne, inventaire 978.23.2978

5. Voir http://www.collections.musee-bretagne.fr/ark:/83011/FLMjo149337 [lien valide en octobre 2021]. Lettre du 11 septembre 1899, collection musée de Bretagne, inventaire 978.23.2046 6. Voir http://www.collections.musee-bretagne.fr/ark:/83011/FLMjo149376 [lien valide en octobre 2021]. Lettre du 13 novembre 1898, collection musée de Bretagne, inventaire 978.23.2057 7. Voir http://www.collections.musee-bretagne.fr/ark:/83011/FLMjo193388 [lien valide en octobre 2021]. Lettre du 4 juillet 1899 de L. Kirmse (Bournemouth), collection musée de Bretagne, inventaire 978.23.21

8. Voir http://www.collections.musee-bretagne.fr/ark:/83011/FLMjo149474 [lien valide en octobre 2021]. Lettre du 11 septembre 1899, collection musée de Bretagne, inventaire 978.23.2115.2

9. ROGÈS Louis \& GERSCHEL Aaron (photographe), L'Affaire Dreyfus. Cinq semaines à Rennes, Paris, F. Juven, 1899, p. 12.

10. Voir la photographie de Pierre Gaigneux à son sujet via http://www.collections.museebretagne.fr/ark:/83011/FLMjo413638 [lien valide en octobre 2021].

11. Hélène Jégado était une prostituée, domestique et cuisinière accusée d'empoisonner des personnes avec lesquelles elle était en contact dans le cadre de son travail. Elle a été guillotinée en 1852.

12. En découvrir plus via http://www.collections.musee-bretagne.fr/ [lien valide en octobre 2021]. 


\section{RÉSUMÉS}

Si le terme de "justice", entendu dans un sens large, évoquant à la fois les hommes qui l'exercent, ceux qui la subissent ou encore les lieux où elle se pratique, alors cette définition plurielle s'incarne du XVII ${ }^{\mathrm{e}}$ siècle à nos jours à travers les collections du musée de Bretagne.

La première collection qui vient immédiatement à l'esprit est celle constituée depuis 1899 autour de l'affaire Dreyfus et spécifiquement du procès de Rennes : ce fonds regroupe aujourd'hui plus de 7000 items, répartis entre de la correspondance, des photographies originales, des cartes postales, des affiches... récemment sont arrivés 13 volumes constitués par le procureur Félix Froissart, lesquels feront l'objet en 2020 d'un travail de recherche en master d'histoire accompagné d'une bourse.

Plus lointaines mais également conséquentes et associées à l'histoire de la ville et de la région, les collections évoquant le parlement de Bretagne: estampes, dessins, photographies, sacs de procédure, portraits de parlementaires, sceaux... Quelques affaires spectaculaires, comme les crimes perpétués par Hélène Jégado sont présentes au musée par le biais d'images populaires ou encore dans le cas présent de son masque mortuaire. Les bâtiments liés à la pratique de la justice, prison centrale des femmes de Rennes ou bagne de Brest sont bien représentés par des plans et des photographies originales du XIX ${ }^{\mathrm{e}}$ siècle.

Les collections témoignent aussi d'une histoire plus récente, par le biais de fonds comme celui de l'association des combattants volontaires de la Résistance, ou encore d'une série d'affiches provenant de mouvements indépendantistes. La particularité de toutes ces collections réside également dans leur libre accès et libre utilisation via le portail des collections : www.collections.musee-bretagne.fr [lien valide en janvier 2022].

If we take the word justice to mean the men and women who practise it, those who endure its decisions and even the places where it is exercised, then it is that multi-dimensional idea which can be observed in the Museum of Brittany's collections from the $17^{\text {th }}$ century to today.

The first collection which comes to mind is that which was started in 1899 around the famous Dreyfus Affair and more precisely the trial which took place in Rennes. This collection currently consists of more than 7.000 items including letters, original photographs, postcards and posters. The museum recently acquired thirteen volumes to enrich its collection, put together by Félix Froissart, the public prosecutor at the time; they will be the object of extensive research via a Master's in History supported by a grant.

If we go back further into the history of the town and the region, the collection has items linked to the Breton Parliament: etchings, drawings, photographs, petty bags, portraits of political figures, rubber stamps and seals. Different popular images illustrate several sensational cases in the museum's collections. One such case is that of Hélène Jégado whose funeral mask is in the vaults. Buildings linked to the theme of justice are represented with plans and original $19^{\text {th }}$ century photos, like those of the women's prisons in Rennes or the jail in Brest where forced labour was endured.

The collections also bear witness to modern history with items linked to the association of voluntary fighters of the Resistance movement and a series of posters from different separatist movements.

These extensive collections come with an added premium: they are all freely accessible via the museum's online collection. 
INDEX

Keywords : justice, Brittany, Dreyfus (Alfred), trial, Rennes, collection, innocence, miscarriage of justice

Mots-clés : justice, Bretagne, Dreyfus (Alfred), procès, Rennes, collection, innocence, erreur judiciaire

\section{AUTEUR}

\section{LAURENCE PROD'HOMME}

musée de Bretagne, Rennes, conservatrice du patrimoine, responsable cellule recherche

l.prodhomme@leschampslibres.fr 